


\title{
Entropy and Reduced Distance for Ricci Expanders
}

\author{
By Michael Feldman, Tom Ilmanen, and Lei Ni
}

\begin{abstract}
Perelman has discovered two integral quantities, the shrinker entropy $\mathcal{W}$ and the (backward) reduced volume, that are monotone under the Ricci flow $\partial g_{i j} / \partial t=-2 R_{i j}$ and constant on shrinking solitons. Tweaking some signs, we find similar formulae corresponding to the expanding case. The expanding entropy $\mathcal{W}_{+}$is monotone on any compact Ricci flow and constant precisely on expanders; as in Perelman, it follows from a differential inequality for a Harnack-like quantity for the conjugate heat equation, and leads to functionals $\mu_{+}$and $v_{+}$. The forward reduced volume $\theta_{+}$is monotone in general and constant exactly on expanders.

A natural conjecture asserts that $g(t) / t$ converges as $t \rightarrow \infty$ to a negative Einstein manifold in some weak sense (in particular ignoring collapsing parts). If the limit is known a-priori to be smooth and compact, this statement follows easily from any monotone quantity that is constant on expanders; these include $\operatorname{vol}(g) / t^{n / 2}$ (Hamilton) and $\bar{\lambda}$ (Perelman), as well as our new quantities. In general, we show that, if $\operatorname{vol}(g)$ grows like $t^{n / 2}$ (maximal volume growth) then $\mathcal{W}_{+}, \theta_{+}$and $\bar{\lambda}$ remain bounded (in their appropriate ways) for all time. We attempt a sharp formulation of the conjecture.
\end{abstract}

Small, large and distant parts of a Ricci flow are known to be modeled by various kinds of Ricci solitons: Steady, shrinking, and expanding. Perelman has discovered monotone quantities corresponding to the first two of these. The functional $\mathcal{F}$ is monotone on the Ricci flow and constant precisely on steadies [25, Section 1]. The shrinking, or localizing, entropy $\mathcal{W}$ is monotone in general and constant precisely on shrinking solitons [25, Section 3]. Closely related is the notion of the (backward) reduced volume [25, Section 7]. The latter two show that developing singularities and ancient histories are modeled on shrinkers, when an appropriate blowup, or blowdown is taken $[25,27]$.

In this note, by tweaking some signs, we observe similar monotonicity formulae for the expanding case. In Section 1, analogous to [25, Section 1], we define an expanding, or delocalizing entropy $\mathcal{W}_{+}$, which is monotone in general and constant precisely on expanders. It follows from

\footnotetext{
Math Subject Classifications. Primary: 58G11.

Key Words and Phrases. Entropy, Ricci flow, Ricci expanders, asymptotic behavior, reduced volume, monotonicity, Li-Yau-Hamilton inequality.

Acknowledgements and Notes. First author was partially supported by NSF grant DMS-0200644; second author was partially supported by Schweizerische Nationalfonds grant 21-66743.01; third author was partially supported by NSF grants and an Alfred P. Sloan Fellowship.
}

(1) 2005 The Journal of Geometric Analysis ISSN 1050-6926 
a differential inequality for the conjugate heat equation similar to [25, Section 9]; see also [20]. We also construct functionals $\mu_{+}(g, \sigma)$ and $\nu_{+}(g)$ from $\mathcal{W}_{+}$.

In Section 2, analogous to [25, Section 7], we present a forward reduced distance $\ell_{+}$and its corresponding forward reduced volume, which is again monotone in general, and constant exactly on expanders.

Our formulae may be compared to those for $\operatorname{vol}(g) / t^{n / 2}$ (Hamilton) and of $\bar{\lambda}(g):=\operatorname{vol}(g)^{2 / n}$ $\lambda(g)$ (Perelman). All of these monotonicities have implications for the convergence of the rescaled Ricci flow as $t \rightarrow \infty$. If $g(t)$ is a compact Ricci flow that exists for all $t>0$, and the volume $\operatorname{vol}(g)$ grows like $t^{n / 2}$ (maximal volume growth), we observe in Proposition 1.4 that $\mathcal{W}_{+}$remains bounded. A similar result holds for Perelman's scaled $\lambda$-functional, and for the forward reduced volume (Proposition 2.3). This suggests that (ignoring zero-volume collapsing parts) the longterm limit of $g(t) / t$ is a negative Einstein manifold, possibly with singularities; see Conjecture 1.6.

Our results may be set against recently discovered entropy formulae and pointwise differential inequalities in other settings: For the standard heat equation on a manifold of non negative Ricci curvature [22], and for the forward conjugate heat equation $u_{t}=\Delta u+R u$ on a two-dimensional Ricci flow [23] and (matrix Harnack!) on a Kähler-Ricci flow [24]. ${ }^{1}$

It is also instructive to compare the Ricci flow to the mean curvature flow in Euclidean space. The monotonicity formulae for $\mathcal{F}, \mathcal{W}, \mathcal{W}_{+}$, and for the corresponding reduced volumes, are analogous to the following three monotone energies on a mean curvature flow $M_{t}$ in $\mathbb{R}^{N}$ :

$$
\begin{aligned}
& \frac{d}{d t} \int_{M_{t}} \frac{e^{-\left|x-x_{0}\right|^{2} / 4\left(t_{0}-t\right)}}{(-4 \pi t)^{n / 2}} \leq 0, \quad t<0, \\
& \frac{d}{d t} \int_{M_{t}} e^{\left\langle x, x_{0}\right\rangle-\left|x_{0}\right|^{2} t} \leq 0, \quad t \in \mathbb{R}, \quad \frac{d}{d t} \int_{M_{t}} \frac{e^{\left|x-x_{0}\right|^{2} / 4\left(t-t_{0}\right)}}{(4 \pi t)^{n / 2}} \leq 0, \quad t>0 .
\end{aligned}
$$

The first is Huisken's well-known result [15] and is constant precisely on shrinkers. The second picks out steadies (translators) [16], whereas the third is stationary on expanders [19].

In the non compact case, for both flows, the energy of a steady, or expander is typically infinite, so in this case the monotonicity formulae will not be useful in proving convergence to an asymptotic soliton. ${ }^{2}$ This is unfortunate, because non compact expanders are needed for modeling the relaxation out of a previously formed singularity of conic type; see, for example, $[2,18,10]$ and remarks after Proposition 2.3.

In the compact case, a sharp disanalogy between the two flows arises. Every compact mean curvature flow disappears in finite time, so mean curvature flow has no compact steadies, or expanders. On the other hand, in Ricci flow, negative curvature allows a compact solution to last forever, even grow. Therefore, compact steadies and expanders exist, but on sufferance: They are always Einstein [13, Section 2]; [8, Proposition 5.20].

On the other hand, shrinkers typically have finite energy whether compact, or non compact. Furthermore, the soliton potential need not be constant. (So a Ricci shrinker $g(t)$ need not be Einstein, and a mean curvature shrinker $M_{t}$ need not be a minimal submanifold of a sphere.) There is accordingly a lively variational calculus for them (see $[15,17,19,30,4]$ and others).

\footnotetext{
${ }^{1}$ It is conjugate to the backward heat equation $u_{t}=-\Delta u$.

${ }^{2}$ There are graphs over $\mathbb{R}^{n}$ (due to Ecker) that evolve by mean curvature flow forever, but are asymptotic neither to expanders nor translators. There also appear to be non compact curves in $\mathbb{R}^{2}$ whose mean curvature flow exhibits non trivial periodicity, i.e., breathers $[3,19]$.
} 


\section{Expander entropy formula}

Let $(M, g(t))$ be a solution to the Ricci flow

$$
\frac{\partial g_{i j}}{\partial t}=-2 R_{i j}
$$

We call $g(t)$ a (gradient) expander, if

$$
g(t)=a(t) \phi_{t}^{*}\left(g_{0}\right)
$$

where $\phi_{t}$ is a family of diffeomorphisms whose velocity is given by a gradient vector field, and $a(t)$ is any increasing function. Then $a(t)=t-T$ for some $T$ and $g$ solves the PDE

$$
R c+\nabla^{2} F+\frac{g}{2(t-T)}=0, \quad t>T
$$

on each time slice, for some function $F=F(\cdot, t)$.

Now let $M$ be closed, $g$ a metric, $u>0$ a function, and $\sigma>0$. Dual to the shrinker entropy $\mathcal{W}\left[25\right.$, Section 3], (which we call $\mathcal{W}_{-}$) define the expander entropy by

$$
\begin{aligned}
\mathcal{W}_{+}(g, u, \sigma) & :=\int_{M}\left[\sigma\left(\frac{|\nabla u|^{2}}{u}+R u\right)+u \log u\right] d v+\frac{n}{2} \log (4 \pi \sigma)+n \\
& =\int_{M}\left[\sigma\left(\left|\nabla f_{+}\right|^{2}+R\right)-f_{+}+n\right] u d v
\end{aligned}
$$

restricted to $u$ satisfying

$$
\int_{M} u d v=1
$$

Here $f_{+}=f_{+}^{u, \sigma}$ is defined via

$$
u=\frac{e^{-f_{+}}}{(4 \pi \sigma)^{n / 2}}
$$

Now take $u=u(x, t)$ to be a positive solution to the conjugate heat equation

$$
\frac{\partial u}{\partial t}=-\Delta u+R u
$$

satisfying the conserved condition $\int u d v=1$. Note that $f_{+}=f_{+}^{u, t-T}$ solves

$$
\frac{\partial f_{+}}{\partial t}=-\Delta f_{+}+\left|\nabla f_{+}\right|^{2}-R-\frac{n}{2(t-T)} .
$$

The following monotonicity formula for $\mathcal{W}_{+}$was found by varying the signs in the shrinker entropy formula [25, Section 3]. A more elegant derivation is given a few pages hence.

Theorem 1.1 (Expander entropy monotonicity). $\quad$ Under evolution Equations (1.1), (1.3) we have

$$
\frac{\partial}{\partial t} \mathcal{W}_{+}(g(t), u(t), t-T)=\int_{M} 2(t-T) u\left|R_{i j}+\nabla_{i} \nabla_{j} f_{+}+\frac{g_{i j}}{2(t-T)}\right|^{2} d v
$$

Note that the right-hand side vanishes precisely on an expander with birth time $T$. 
Formula (1.4) is a consequence of the following differential inequality, which is similar to the one in [25, Section 9] (see also [20]), but with sign changes.

Theorem 1.2. Let $(M, g(t)), u, f_{+}$, and $T$ be as above. Define

$$
v_{+}:=\left[(t-T)\left(2 \Delta f_{+}-\left|\nabla f_{+}\right|^{2}+R\right)-f_{+}+n\right] u, \quad t>T .
$$

Then

$$
\left(\frac{\partial}{\partial t}+\Delta-R\right) v_{+}=2(t-T) u\left|R_{i j}+\nabla_{i} \nabla_{j} f_{+}+\frac{g_{i j}}{2(t-T)}\right|^{2}, \quad t>T .
$$

Proof. We may assume $T=0$. Set $V:=2 \Delta f_{+}-\left|\nabla f_{+}\right|^{2}+R$ and compute

$$
\left(\frac{\partial}{\partial t}+\Delta\right) V=2\left|R_{i j}+\nabla_{i} \nabla_{j} f_{+}\right|^{2}+2\left\langle\nabla V, \nabla f_{+}\right\rangle
$$

and

$$
\left(\frac{\partial}{\partial t}+\Delta\right)\left(t V-f_{+}+n\right)=2 t\left|R_{i j}+\nabla_{i} \nabla_{j} f_{+}\right|^{2}+2\left\langle\nabla\left(t V-f_{+}+n\right), \nabla f_{+}\right\rangle+2 \Delta f_{+}+2 R+\frac{n}{2 t}
$$

and do further calculation.

Proof of Theorem 1.1. Note that $\int v_{+} d v=\mathcal{W}_{+}$. To get the time derivative of $\mathcal{W}_{+}$, integrate (1.5) over $M$.

Remark. For the steady case, one can define $e^{-f}=u, v_{0}:=V u=\left(2 \Delta f-|\nabla f|^{2}+R\right) u$ and verify

$$
\left(\frac{\partial}{\partial t}+\Delta-R\right) v_{0}=2 u\left|R_{i j}+\nabla_{i} \nabla_{j} f\right|^{2},
$$

which may be viewed as a "neutral" differential Harnack inequality. It may be integrated to get the monotonicity of $\mathcal{F}$ in [25, Section 1]. See [7].

We next consider the implications for long term behavior of the Ricci flow. Let $M$ be compact and consider a Ricci flow $g(t)$ that exists for all $t>b$. We construct a global solution of the conjugate heat equation. Let $t^{i} \rightarrow \infty$, and let $u^{i}, b<t \leq t^{i}$ be the solution of (1.3) with final data

$$
u^{i}\left(x, t^{i}\right)=\frac{1}{V\left(t^{i}\right)}, \quad x \in M
$$

Passing $t^{i} \rightarrow \infty$ we obtain an immortal solution $u>0$ of (1.3) with $\int_{M} u=1$ for all $t>b$.

Now let $\alpha_{j} \rightarrow \infty$ and define the blowdown sequence

$$
g_{j}(t):=\alpha_{j}^{-1} g\left(\alpha_{j} t\right), \quad t>b / \alpha_{j} .
$$

The following theorem is classic. We give a new proof using $\mathcal{W}_{+}$. The hypothesis will hold (subsequentially) if, for example, $|R m|$, diameter, and injectivity radius of $g(t) / t$ are controlled for all time [12]. A similar theorem has been proven for the shrinking case [27].

Theorem 1.3 (Hamilton [13]). Let $M$ be compact. Suppose there are diffeomorphisms $\phi_{j}$ such that $\phi_{j}^{*}\left(g_{j}(t)\right) \rightarrow g_{\infty}(t)$ smoothly, $t>0$. Then $g_{\infty}(t)$ is an expanding soliton (hence negative Einstein). 
Proof. Let $u_{j}(t):=\alpha_{j}^{-n / 2} u\left(\alpha_{j} t\right)$. Evidently we can extract a smoothly convergent subsequence $\phi_{j}^{*}\left(u_{j}\right) \rightarrow u_{\infty}>0$ satisfying (1.3) for all time. The convergence is smooth, the manifold is compact, and $\mathcal{W}_{+}$is monotone and scale invariant. So $\mathcal{W}_{+}$is constant on the limit flow, which is therefore an expanding soliton.

Remark. Note that an expanding breather can be expressed as a blowdown of itself, after extending it iteratively to a maximal interval of existence $t>b$. Applying the theorem then yields an alternative proof that there are no non trivial compact expanding breathers [25, Section 2].

Theorem 1.3 may be proven using any monotone, scale invariant quantity that is constant only on expanders. Hamilton's original method [13] uses the quantity $R_{\min }(t) V(t)^{2 / n}$. Alternately (Hamilton), one can use the scaled volume

$$
\tilde{V}(t):=\frac{V(t)}{t^{n / 2}} .
$$

Here $V(t)$ is the volume of $g(t)$ and $\tilde{V}(t)$ is the volume of the rescaled metric $\tilde{g}(t):=g(t) / t$. Perelman's proof [25, Section 2] uses the scaled $\lambda$-functional

$$
\bar{\lambda}(g):=V(g)^{2 / n} \lambda(g)
$$

where $\lambda(g):=\inf \left\{\mathcal{F}(g, u) \mid \int u d v=1\right\}$. One can also use the forward reduced volume (see Section 2). In each case, the right-hand side of the monotonicity formula vanishes identically only on expanders.

We now drop the hypothesis that the blowdowns converge smoothly to a compact limit. Can we still get convergence to expanders? The various monotonicity formulae will only be useful, if the monotone quantity remains bounded for all time. Accordingly, we embark on a study of the long-term behavior of $\mathcal{W}_{+}, \tilde{V}$, and $\bar{\lambda}$.

We begin with the scaled volume. Recall from Hamilton [13]

$$
\frac{\partial R}{\partial t}=\Delta R+2|R c|^{2}
$$

from which follows via $R^{2} \leq n|R c|^{2}$ and the maximum principle that

$$
R+\frac{n}{2 t} \geq 0, \quad \frac{d \tilde{V}}{d t}=-\frac{1}{t^{n / 2}} \int\left(R+\frac{n}{2 t}\right) d v \leq 0 .
$$

So $\tilde{V}(t)$ is non increasing, and $\tilde{V}_{\infty}:=\lim _{t \rightarrow \infty} \tilde{V}(t)$ exists. $^{3}$

Next we motivate $\mathcal{W}_{+}$as mentioned above. Analogously to [25, Section 5] (see also [23]), define

$$
\mathcal{N}(g, u):=\int_{M} u \log u d v, \quad \mathcal{N}_{+}(g, u, \sigma):=\mathcal{N}(g, u)+\frac{n}{2} \log (4 \pi \sigma)+\frac{n}{2} .
$$

\footnotetext{
${ }^{3}$ The proof of Theorem 1.3 (Hamilton) then runs as follows: Assume $g_{j}(t)$ converges smoothly to a compact limit $g_{\infty}(t)$. Then $\tilde{V}(g(t)) \rightarrow \tilde{V}\left(g_{\infty}(t)\right)$, which is therefore constant, so $R_{\infty} \equiv-n / 2 t$, so $R c_{\infty}$ is diagonal and equals $-g_{\infty} / 2 t$. This shows that any such blowdown is negative Einstein. In particular, any compact expander is negative Einstein.
} 
The quantity $\mathcal{N}$ is the Nash entropy [21]. ${ }^{4}$ Note that $\mathcal{N}_{+}$is dual to the entropy used for shrinkers in [25, Section 5]. Now let $g(t)$ and $u(t)$ evolve as above for all $t>0$ and set $\sigma(t):=t$. Compute

$$
\frac{d \mathcal{N}}{d t}=\mathcal{F}:=\int_{M}\left(\frac{|\nabla u|^{2}}{u}+R u\right) d v, \quad \frac{d \mathcal{N}_{+}}{d t}=\mathcal{F}_{+}:=\mathcal{F}+\frac{n}{2 t},
$$

and

$$
\begin{aligned}
\frac{\partial}{\partial t}\left(t \mathcal{N}_{+}\right) & =\mathcal{N}_{+}+t \mathcal{F}_{+} \\
& =\int u\left[\log u+\frac{n}{2} \log (4 \pi t)+\frac{n}{2}+\frac{n}{2}+t\left(\frac{|\nabla u|^{2}}{u}+R u\right)\right] d v \\
& =\mathcal{W}_{+},
\end{aligned}
$$

which motivates $\mathcal{W}_{+}$.

Next we recall [25, Section 2]

$$
\frac{d}{d t} \mathcal{F} \geq \frac{2}{n} \mathcal{F}^{2}
$$

By ODE comparison, if $g(t)$ exists for all $t>0$ we have

$$
-\frac{n}{2 t} \leq \mathcal{F}(g, u) \leq 0, \quad t>0 .
$$

(Side remark: Passing the starting time to $-\infty$, we notice that a compact eternal solution has $\mathcal{F} \equiv 0$, hence is a steady soliton. Namely, any compact eternal solution must be a steady soliton. ${ }^{5}$ )

Next we study $\mathcal{N}, \mathcal{W}_{+}$, and $\bar{\lambda}$ as $t \rightarrow \infty$. By Jensen's inequality, we have in general

$$
\int u \log u d v \geq \log \left(\frac{1}{V}\right)
$$

when $\int u d v=1$, with equality precisely when $u=1 / V$. So we have the lower bound

$$
\mathcal{N}_{+}(g, u, t) \geq-\log (V(t))+\frac{n}{2} \log (4 \pi t)+\frac{n}{2} .
$$

To get a similar upper bound, we use monotonicity and the construction of $u$ as asymptotically constant. From above we have

$$
\frac{d}{d t} \mathcal{N}_{+}(g, u, t)=\mathcal{F}_{+}(g, u, t)=\mathcal{F}(g, u)+\frac{n}{2 t} \geq 0,
$$

so $\mathcal{N}_{+}$is monotone non decreasing. This also applies also to $u^{i}$. So fixing $t$, we have for all large $t^{i}$,

$$
\mathcal{N}_{+}\left(g, u^{i}, t\right) \leq \mathcal{N}_{+}\left(g, u^{i}, t^{i}\right)=\log \left(\frac{1}{\operatorname{Vbig}\left(t^{i}\right)}\right)+\frac{n}{2} \log \left(4 \pi t^{i}\right)+\frac{n}{2} .
$$

Taking $i \rightarrow \infty$, combining with the lower bound, and sending $t \rightarrow \infty$, we obtain

$$
\lim _{t \rightarrow \infty} \mathcal{N}_{+}(g, u, t)=-\log \left(\tilde{V}_{\infty}\right)+\frac{n}{2}(\log 4 \pi+1) .
$$

\footnotetext{
${ }^{4}$ Our sign convention for $\mathcal{N}$ is the opposite of Nash's.

${ }^{5}$ Very recently, a similar result has been proved in [9] for non compact, two-dimensional eternal solutions, under an assumption on the asymptotic behavior of the conformal factor.
} 
(This holds even, if $\tilde{V}_{\infty}=0$.) If $\tilde{V}_{\infty}>0$, then since $\mathcal{N}_{+}$is monotone, there exist $t_{j} \rightarrow \infty$ such that

$$
\lim _{j \rightarrow \infty} t_{j} \mathcal{F}_{+}\left(g, u, t_{j}\right)=0 .
$$

Since $\mathcal{W}_{+}=t \mathcal{F}_{+}+\mathcal{N}_{+}$, and since $\mathcal{W}_{+}$is monotone, we obtain

$$
\lim _{t \rightarrow \infty} \mathcal{W}_{+}(g, u, t)=\lim _{t \rightarrow \infty} \mathcal{N}_{+}(g, u, t)
$$

(Again, it holds even, if $\tilde{V}_{\infty}=0$.)

Next we turn to $\bar{\lambda}$. It is straightforward to show that the minimizer in the definition of $\lambda$ depends smoothly on $g$, so $\lambda(g(t))$ is smooth, and

$$
\frac{d \lambda}{d t} \geq \frac{2}{n} \lambda^{2}, \quad-\frac{n}{2} \leq t \lambda \leq 0
$$

since $g(t)$ exists for all $t>0$. From $t_{j} \mathcal{F}_{+} \rightarrow 0$ and the lower bound for $\lambda$ we get

$$
\lim _{t_{j} \rightarrow \infty} t_{j} \lambda(g)=\lim _{t_{j} \rightarrow \infty} t_{j} \mathcal{F}(g, u)=-\frac{n}{2} .
$$

Using $\lambda \leq \int R d v / V$, (1.7), and the fact that $\lambda \leq 0$, we obtain Perelman's monotonicity [25, Section 2],

$$
\frac{d}{d t} \bar{\lambda}(g(t)) \geq 0
$$

Since $t^{-1} V(t)^{2 / n} \rightarrow \tilde{V}_{\infty}^{2 / n}$ we obtain

$$
\lim _{t \rightarrow \infty} \bar{\lambda}(g)=-\frac{n}{2} \tilde{V}_{\infty}^{n / 2}
$$

Our results are summarized in the following proposition, which by inspecting the above argument holds even, if $\tilde{V}_{\infty}=0$.

Proposition 1.4. Let $M$ be compact, $g(t)$ a Ricci flow for all $t \geq 0$, and $u(t)$ the solution of the conjugate heat equation constructed above. Then

$$
\lim _{t \rightarrow \infty} \mathcal{W}_{+}(g(t), u(t), t)=-\log \tilde{V}_{\infty}+\frac{n}{2}(1+\log 4 \pi)
$$

and

$$
\lim _{t \rightarrow \infty} \bar{\lambda}(g(t))=-\frac{n}{2} \tilde{V}_{\infty}^{2 / n}
$$

Remark. The upper bound on $\mathcal{W}_{+}$can be proven without using $\mathcal{N}_{+}$. If $\tilde{V}_{\infty}>0$ then from the monotonicity of $t^{-n / 2} V(t)$ we get the estimate

$$
0 \leq \int_{0}^{\infty} t^{-n / 2} \int_{M}\left(R+\frac{n}{2 t}\right) d v d t<\infty .
$$

So there exist $t^{i} \rightarrow \infty$ so that

$$
\left(t^{i}\right)^{-n / 2} \int t^{i} R d v \rightarrow-\frac{n}{2} \tilde{V}_{\infty} .
$$


Employ this particular sequence $t^{i}$ in the construction of $u$ (it should not affect the value of $u$, but we have not proven this) yields for each fixed $t$ and large $t^{i}$,

$$
\begin{aligned}
\mathcal{W}_{+}\left(g(t), u^{i}(t), t\right) & \leq \mathcal{W}_{+}\left(g\left(t^{i}\right), u^{i}\left(t^{i}\right), t^{i}\right) \\
& =\frac{1}{V\left(t^{i}\right)} \int t^{i} R d v-\log V\left(t^{i}\right)+\frac{n}{2} \log \left(4 \pi t^{i}\right)+n \\
& \rightarrow-\log \tilde{V}_{\infty}+\frac{n}{2}(1+\log (4 \pi)), \quad t^{i} \rightarrow \infty
\end{aligned}
$$

The result follows by passing $u^{i} \rightarrow u$.

As a corollary to Proposition 1.4, we obtain the following estimate.

Proposition 1.5. Let $M$ be compact and let $g(t), u(t)$ solve (1.1), (1.3) for all $t>0$. Assume $\tilde{V}_{\infty}>0$. Then the rescaled solution $\tilde{g}(\tilde{t}):=g(t) / t, \tilde{u}(\tilde{t}):=t^{n / 2} u, \tilde{f}_{+}(\tilde{t}):=f_{+}(t), \tilde{t}=\log t$ satisfies

$$
\int_{0}^{\infty} \int_{M} \tilde{u}\left|\tilde{R} c+\tilde{\nabla}^{2} \tilde{f}_{+}+\frac{\tilde{g}}{2}\right|^{2} d \tilde{v} d \tilde{t}<\infty
$$

This estimate may provide enough control to eventually deduce convergence of a blowdown sequence in a suitable weak sense. We formulate the following natural conjecture as sharply as possible.

Conjecture 1.6 (Long-term behavior). Let $g(t)$ be a Ricci flow that exists for all $t>0$ on a compact manifold $M$. Then there is an open set $U \subset M$, times $t_{j} \rightarrow \infty$, and a metric $\tilde{g}_{\infty}$ such that

$$
g\left(t_{j}\right) / t_{j} \rightarrow \tilde{g}_{\infty} \quad \text { in } U,
$$

where $\tilde{g}_{\infty}$ is smooth and negative Einstein, the metric completion of $\tilde{g}_{\infty}$ has a singular set of dimension at most $n-4$, and

$$
\operatorname{vol}\left(\tilde{g}_{\infty}\right)=\tilde{V}_{\infty}
$$

The metric completion of $\tilde{g}_{\infty}$ need not be compact, or connected, but has finite volume, so it should be negative Einstein and not merely an expander. The set $U$ need not be dense in $M$. If $\tilde{V}_{\infty}=0$, then take $U$ to be empty and the statement holds vacuously; more generally, the assertion is that the scaled volume of the collapsing parts tends to zero. The rescaling is designed not to see these parts: They recede to infinity, or hide beyond the singular set.

The conjecture is inspired by the convergence picture in the three-dimensional case [13, 26]; a good exercise would be to employ the $\mathcal{W}_{+}$monotonicity as an alternative way of recognizing the hyperbolic pieces in that case. The codimension of the singular set is motivated by the fact that the right-hand side $\int u|R m+\cdots|^{2}$ is unitless for $n=4$, and is supported by familiar convergence results for Einstein 4-manifolds ([1, 5, 6, 14, 27, 28, 29] and many others), as well as analogous results for mean curvature flow in the critical case [17] and other flows. Later Professor Tian informed us that he had independently formulated a similar conjecture.

Formulation of $\mu_{+}$and $v_{+}$functionals. Define, analogously to [25, Section 3],

$$
\mu_{+}(g, \sigma):=\inf _{u} \mathcal{W}_{+}(g, u, \sigma), \quad v_{+}(g):=\sup _{\sigma>0} \mu_{+}(g, \sigma),
$$


where $u$ varies over functions satisfying $\int_{M} u d v=1$. We investigate the existence, smoothness, and monotonicity of these quantities.

Theorem 1.7. Let $M$ be a closed manifold.

(a) The inf in the definition of $\mu_{+}$is attained by a unique $u . \mu_{+}(g(t), t-T)$ is monotone non decreasing under the Ricci flow, and is constant only on an expander with starting time $t=T$.

(b) If $\lambda(g)<0$, then the sup in the definition of $v_{+}$is attained by a unique $\sigma \cdot v_{+}(g(t))$ is monotone non decreasing under the Ricci flow, and is constant only on an expander.

Proof. (a) Write

$$
\mathcal{W}_{+}=\int\left[\sigma\left(4|\nabla w|^{2}+R w^{2}\right)+w^{2} \log w^{2}\right] d v+C,
$$

where $w^{2}=u$. This functional is lower semicontinous and coercive on $W^{1,2}$, so it possesses a minimizer $w$ subject to $\int w^{2}=1$. Without loss of generality, $w$ is non negative. By the strong maximum principle (taking into account the sign of the nonlinearity), $w>0$ and therefore $w$ is smooth. The corresponding $u=w^{2}$ minimizes the strictly convex functional

$$
\mathcal{W}_{+}=\int\left[\sigma\left(4\left|\nabla u^{1 / 2}\right|^{2}+R u\right)+u \log u\right] d v+C
$$

on the cone of smooth functions with $u>0, \int u=1$, and therefore $u$ is unique. It is straightforward to verify that $u$ varies smoothly when $(g, \sigma)$ varies smoothly. Write $u_{g, \sigma}$ for this minimizer.

Now let $g(t)$ be a Ricci flow, fix $T \in \mathbb{R}$, and let $\tilde{u}(t):=u_{g(t), t-T}$ be the minimizer for each $t$. Fix $t_{0}>T$ and let $u(t) t \leq t_{0}$ be the solution of the conjugate heat Equation (1.3) with final value $u\left(t_{0}\right)$. Since the first variation of $\mathcal{W}_{+}$at $t=t_{0}$ with respect to $u$ vanishes, we can compute (smoothly) at $t=t_{0}$

$$
\frac{d}{d t} \mu_{+}(g(t), t-T)=\frac{d}{d t} \mathcal{W}_{+}(g(t), \tilde{u}(t), t-T)=\frac{d}{d t} \mathcal{W}_{+}(g(t), u(t), t-T),
$$

so

$$
\frac{d}{d t} \mu_{+}(g(t), t-T)=\int u\left|R c+\nabla^{2} f_{+}+\frac{g}{2(T-t)}\right|^{2} d v,
$$

where $u$ realizes the minimum at $t=t_{0}$. Since $t_{0}$ was arbitrary, this formula actually holds for each $t$, where $u$ realizes the minimum at time $t$. If $\mu_{+}(g(t), t-T)$ is constant on any interval, the right-hand vanishes, so $g(t)$ is an expander with starting time $T$.

(b) By the above, $\mu_{+}(g, \sigma)$ is continuous in $\sigma, \sigma>0$. We show that it goes to $-\infty$ at the endpoints. Recall [25, Section 1]

$$
\mathcal{F}(g, u):=\int \frac{|\nabla u|^{2}}{u}+R u d v, \quad \lambda(g):=\inf _{u} \mathcal{F}(g, w),
$$

where $u$ ranges over functions with $\int u=1$. Fix $u$ and estimate

$$
\mu_{+}(g, \sigma) \leq \mathcal{W}_{+}(g, u, \sigma)=\sigma \mathcal{F}(g, u)+\int u \log u d v+\frac{n}{2} \log (4 \pi \sigma)+n .
$$

Thus, $\mu_{+}(g, \sigma) \rightarrow-\infty$ as $\sigma \rightarrow 0$. If $\lambda(g)<0$, we select $u$ so that $\mathcal{F}(g, u)=\lambda(u)$ and find that $\mu_{+}(g, \sigma) \rightarrow-\infty$ as $\sigma \rightarrow \infty$. In addition it is strictly concave in $\sigma$. Therefore the supremum in $\nu_{+}$is realized by some unique $\sigma=\sigma_{g}$. Evidently $\sigma_{g}$ varies smoothly with $g$. 
Now let $g(t)$ be a Ricci flow and fix $t_{0}>0$. Since the first variation of $\mu_{+}\left(g\left(t_{0}\right), \sigma\right)$ is zero at $\sigma=\sigma_{g\left(t_{0}\right)}$, we may compute (smoothly) at $t=t_{0}$

$$
\frac{d}{d t} \nu_{+}(g(t))=\frac{d}{d t} \mu_{+}\left(g(t), \sigma_{g(t)}\right)=\frac{d}{d t} \mu_{+}\left(g(t), \sigma+t-t_{0}\right) .
$$

Thus,

$$
\frac{d}{d t} v_{+}(g(t))=\int u\left|R c+\nabla^{2} f_{+}+\frac{g}{2 \sigma}\right|^{2} d v,
$$

where $(u, \sigma)$ realizes the minimax at $t=t_{0}$. Since $t_{0}$ was arbitrary, this formula actually holds for each $t$, where $(u, \sigma)$ realizes the minimax at time $t$. If $v_{+}(g(t))$ is ever constant, the right-hand vanishes, so $g(t)$ is an expander.

\section{Forward reduced distance and reduced volume}

In this section we derive the dual version of Perelman's monotonicity on the reduced volume in [25, Section 7].

Let $g(t)$ solve the Ricci flow on $M \times[0, T]$. Fix $x_{0}$ and let $\gamma$ be a path $(x(\eta), \eta)$ joining $\left(x_{0}, 0\right)$ to $(y, t)$. Analogous to [25], we define

$$
\mathcal{L}_{+}(\gamma)=\int_{0}^{t} \sqrt{\eta}\left(R+\left|\gamma^{\prime}(\eta)\right|^{2}\right) d \eta
$$

Let $X \equiv \gamma^{\prime}(t)$ and let $Y$ be a variational vector field along $\gamma$. One calculates the first variation of $\mathcal{L}_{+}$to be

$$
\delta \mathcal{L}_{+}=\left.2 \sqrt{t}\langle X, Y\rangle\right|_{0} ^{t}+\int_{0}^{t} \sqrt{\eta}\left\langle Y, \nabla R-2 \nabla_{X} X+4 R c(X, \cdot)-\frac{1}{\eta} X\right\rangle d \eta .
$$

From this one can write the $\mathcal{L}_{+}$-geodesic equation:

$$
\nabla_{X} X-\frac{1}{2} \nabla R+\frac{1}{2 t} X-2 R c(X, \cdot)=0 .
$$

This differs from [25] only by the sign in front of $\operatorname{Rc}(X, \cdot)$. Let $L_{+}(y, t)$ denote the length of a shortest $\mathcal{L}_{+}$-geodesic joining $\left(x_{0}, 0\right)$ to $(y, t)$. From $(2.2)$ we know that

$$
\nabla L_{+}=2 \sqrt{t} X(t) .
$$

Following closely the computation of [25, Section 7] we obtain

$$
\begin{aligned}
\left|\nabla L_{+}\right|^{2} & =-4 t R+4 t\left(R+|X|^{2}\right) \\
\frac{\partial L_{+}}{\partial t} & =2 \sqrt{t} R-\sqrt{t}\left(R+|X|^{2}\right) .
\end{aligned}
$$

A calculation using the geodesic equation shows that

$$
\frac{d}{d t}\left(R+|X|^{2}\right)=H(X)-\frac{1}{t}\left(R+|X|^{2}\right),
$$

where $H(X):=\partial R / \partial t+2\langle\nabla R, X\rangle+2 R c(X, X)+R / t$ is the exactly twice the traced Li-YauHamilton differential Harnack expression in [11]. (Notice that the $H$ in [25, Section 7] also equals the LYH expression, but evaluated at a negative time $t=-\tau$.) This gives

$$
t^{3 / 2}\left(R+|X|^{2}\right)=K+\frac{1}{2} L_{+}
$$


where

$$
K:=\int_{0}^{t} \eta^{3 / 2} H(X) d \eta
$$

We then have

$$
\begin{aligned}
\left|\nabla L_{+}\right|^{2} & =-4 t R+\frac{2}{\sqrt{t}} L_{+}+\frac{4}{\sqrt{t}} K, \\
\frac{\partial L_{+}}{\partial t} & =2 \sqrt{t} R-\frac{1}{t} K-\frac{1}{2 t} L_{+} .
\end{aligned}
$$

These differ from [25] only by the sign in front of $K$ (but recall that the interpretation of $H$ also differs).

We can similarly do the second variation computation. For the a variation vector $Y$ satisfying $Y(0)=0$, we get

$$
\begin{aligned}
\delta_{Y}^{2} \mathcal{L}_{+}= & 2 \sqrt{t}\left\langle\nabla_{Y} Y, X\right\rangle+\int_{0}^{t} \sqrt{\eta}\left(Y \cdot Y \cdot R-2\left\langle\nabla_{Y} Y, \nabla_{X} X\right\rangle\right. \\
& +4 Y(R c(Y, X))-2 X(R c(Y, Y))-2 R(X, Y, X, Y) \\
& \left.+2\left|\nabla_{X} Y\right|^{2}-\frac{1}{\eta}\left\langle\nabla_{Y} Y, X\right\rangle\right) d \eta .
\end{aligned}
$$

Using the $\mathcal{L}_{+}$-geodesic equation we get

$$
\begin{aligned}
\delta_{Y}^{2} \mathcal{L}_{+}= & 2 \sqrt{t}\left\langle\nabla_{Y} Y, X\right\rangle+\int_{0}^{t} \sqrt{\eta}\left(\nabla^{2} R(Y, Y)-2 R(X, Y, X, Y)\right. \\
& \left.+2\left|\nabla_{X} Y\right|^{2}+4 \nabla_{Y} R c(Y, X)-2 \nabla_{X} R c(Y, Y)\right) d \eta
\end{aligned}
$$

We choose $Y$ such that

$$
\nabla_{X} Y=R c(Y, \cdot)+\frac{1}{2 t} Y,
$$

which in particular implies that $|Y(\eta)|^{2}=\frac{\eta}{t}|Y(t)|^{2}$. Then we obtain

$$
\begin{aligned}
\nabla^{2} L_{+}(Y, Y) \leq & \frac{1}{\sqrt{t}}|Y(t)|^{2}+\int_{0}^{t} \sqrt{\eta}\left(\nabla^{2} R(Y, Y)-2 R(X, Y, X, Y)+2|R c(\cdot, Y)|^{2}\right. \\
& \left.+\frac{2}{\eta} \operatorname{Rc}(Y, Y)\left(4 \nabla_{Y} R c(Y, X)-4 \nabla_{X} R c(Y, Y)\right)+2 \nabla_{X} \operatorname{Rc}(Y, Y)\right) d \eta .
\end{aligned}
$$

Now we use the fact that

$$
\frac{d}{d t}(R c(Y, Y))=\frac{\partial R c}{\partial t}(Y, Y)+\nabla_{X} R c(Y, Y)+2|R c(\cdot, Y)|^{2}+\frac{1}{t} R c(Y, Y)
$$

which implies

$$
\begin{aligned}
2 \int_{0}^{t} \sqrt{\eta} \nabla_{X} R c(Y, Y) d \eta= & 2 \sqrt{t} \operatorname{Rc}(Y, Y) \\
& -\int_{0}^{t} \sqrt{\eta}\left(3 \frac{R c(Y, Y)}{\eta}-2 \frac{\partial R c}{\partial t}(Y, Y)-4|R c(Y, \cdot)|^{2}\right) d \eta .
\end{aligned}
$$


Plugging (2.14) into (2.13) we have

$$
\nabla^{2} L_{+}(Y, Y) \leq \frac{|Y|^{2}}{\sqrt{t}}+2 \sqrt{t} \operatorname{Rc}(Y, Y)-\int_{0}^{t} \sqrt{\eta} H(X, Y) d \eta .
$$

Here

$$
\begin{aligned}
H(X, Y):= & -\nabla^{2} R(Y, Y)+2 R(X, Y, X, Y)+2|R c(\cdot, Y)|^{2} \\
& +\frac{1}{t} R c(Y, Y)+2 \frac{\partial R c}{\partial t}(Y, Y) \\
& -4 \nabla_{Y} R c(Y, X)+4 \nabla_{X} R c(Y, Y) .
\end{aligned}
$$

This is exactly twice Hamilton's matrix LYH expression. We get the following.

Corollary 2.1. If $(M, g(t))$ has non negative curvature operator, then

$$
\nabla^{2} L_{+}(Y, Y) \leq \frac{|Y|^{2}}{\sqrt{t}}+2 \sqrt{t} \operatorname{Rc}(Y, Y)
$$

In the general curvature case, tracing (2.15) in $Y$ yields

$$
\Delta L_{+} \leq \frac{n}{\sqrt{t}}+2 \sqrt{t} R-\frac{1}{t} K
$$

Now defining

$$
\ell_{+}(y, t):=\frac{1}{2 \sqrt{t}} L_{+}(y, t), \quad \bar{L}_{+}(y, t):=4 t \ell_{+}(y, t)
$$

we get

$$
\begin{aligned}
\left|\nabla \ell_{+}\right|^{2} & =-R+\frac{\ell_{+}}{t}+\frac{K}{t^{3 / 2}}, \\
\frac{\partial \ell_{+}}{\partial t} & =R-\frac{K}{2 t^{3 / 2}}-\frac{\ell_{+}}{t} \\
\Delta \ell_{+} & \leq R+\frac{n}{2 t}-\frac{K}{2 t^{3 / 2}}
\end{aligned}
$$

These then imply

$$
\begin{aligned}
\frac{\partial \ell_{+}}{\partial t}+\Delta \ell_{+}+\left|\nabla \ell_{+}\right|^{2}-R-\frac{n}{2 t} & \leq 0, \\
\left(\frac{\partial}{\partial t}-\Delta\right)\left(\bar{L}_{+}+2 n t\right) & \geq 0,
\end{aligned}
$$

and negativity of a quantity resembling $-v_{+}$:

$$
t\left(2 \Delta \ell_{+}+\left|\nabla \ell_{+}\right|^{2}-R\right)-\ell_{+}-n \leq 0 .
$$

Note that $\ell_{+}$resembles $-f_{+}$, in contrast to the shrinker case, where $\ell$ is like $f$ [25, Section 9], [4]. We have the following theorem from (2.18), (2.19).

Theorem 2.2. Let $g(t)$ solve the Ricci flow on $M \times[0, T]$. Then

$$
\hat{u}(x, t):=\frac{e^{\ell_{+}(x, t)}}{(4 \pi t)^{n / 2}}
$$


is a super-solution to the conjugate heat equation, that is, $\partial \hat{u} / \partial t+\Delta \hat{u}-R \hat{u} \leq 0$. In particular, when $M$ is compact, ${ }^{6}$ the forward reduced volume

$$
\theta_{+}(t)=\theta_{+}^{\left(x_{0}, 0\right)}(t):=\int_{M} \hat{u} d v
$$

is monotone non increasing along the flow. Furthermore, $\bar{L}_{+}+2 n t$ is a super-solution to the standard heat equation.

Remarkably, the entropies $\mathcal{W}$ and $\mathcal{W}_{+}$both increase in forward time, whereas the reduced volumes increase as $t$ approaches the reference time $t=0$.

The forward reduced volume is a constant precisely when $g(t)$ is a compact expanding soliton and $\left(x_{0}, 0\right)$ is the "vertex" of the soliton. In this case, we get

$$
\log \theta_{+}(t)=-v_{+}(g),
$$

a constant independent of time. Details will be given in future work; see [4] for the shrinker case. The following proposition says that $\theta_{+}$is bounded away from zero when $\tilde{V}_{\infty}>0$, in the same way as the other monotone quantities.

Proposition 2.3. The forward reduced volume is bounded below by

$$
\log \theta_{+}(t) \geq \log \tilde{V}(t)-\frac{n}{2}(1+\log 4 \pi) .
$$

Proof. Since $R \geq-n / 2 t$, we get for any curve $\gamma$ from $\left(x_{0}, 0\right)$ to $(p, t)$,

$$
\mathcal{L}_{+}(\gamma) \geq \int_{0}^{t} \sqrt{\eta}\left(-\frac{n}{2 \eta}\right) d \eta=-n \sqrt{t},
$$

so $\ell_{+}(p, t) \geq-n / 2$ and

$$
\theta_{+}(t) \geq \frac{V(t) e^{-n / 2}}{(4 \pi t)^{n / 2}}=\frac{\tilde{V}(t)}{(4 \pi e)^{n / 2}}
$$

This also shows that $\theta_{+}(t) \rightarrow \infty$ as $t \rightarrow 0$ unless $V(t) \sim t^{n / 2}$ for small $t$ (maximal volume decay). In particular, when the initial metric is smooth except for a conic singularity $x_{0}$, as may arise by evolution from a smooth metric defined for negative times [10], then $\theta_{+}(t)$ will not be useful in proving that a blowup about the vertex $\left(x_{0}, 0\right)$ for small $t>0$ yields an expander.

\section{References}

[1] Anderson, M. T. The $L^{2}$ structure of moduli spaces of Einstein metrics on 4-manifolds, Geom. Funct. Anal. 2, 29-89, (1992).

[2] Angenent, S. B., Chopp, D., and Ilmanen, T. A computed example of nonuniqueness of mean curvature flow in $\mathbb{R}^{3}$, Comm. Part. Diff. Eq. 20, 1937-1958, (1995).

\footnotetext{
${ }^{6}$ A localized reduced volume, as well as a localized monotonicity formula, has been found in [24], which removes this restriction on $M$ being compact.
} 
[3] Barnes, I. and Ilmanen, T. unpublished computer study, (1994).

[4] Cao, H.-D., Hamilton, R. S., and Ilmanen, T. Gaussian densities and stability for some Ricci solitons, math.DG/ 0404165, April (2004).

[5] Cheeger, J. and Colding, T. H. On the structure of spaces with Ricci curvature bounded below. I, J. Differential Geom. 46, 406-480, (1997).

[6] Cheeger, J. and Tian, G. Collapsing and noncollapsing of Einstein 4-manifolds, in preparation, (2004).

[7] Chow, B., Chu, S.-C., Lu, P., and Ni, L. Notes on Perelman's articles on Ricci flow.

[8] Chow, B. and Knopf, D. The Ricci flow, vol. I: An introduction, Math. Surveys and Monographs, AMS 110, (2004).

[9] Daskalopoulos, P. Eternal solutions to the Ricci flow on $\mathbb{R}^{2}$, preprint, (2004).

[10] Feldman, M., Ilmanen, T., and Knopf, D. Rotationally symmetric shrinking and expanding gradient Kähler-Ricci solitons, J. Differential Geom. 65, 169-209, (2003).

[11] Hamilton, R. S. The Harnack estimate for the Ricci flow, J. Differential Geom. 37, 225-243, (1993).

[12] Hamilton, R.S. A compactness property for solutions of the Ricci flow, Amer. J. Math. 117, 545-572, (1995).

[13] Hamilton, R. S. Non-singular solutions of Ricci flow on three-manifolds, Comm. Anal. Geom. 7, 695-729, (1999).

[14] Hong, M.-C. and Tian, G. Asymptotical behavior of the Yang-Mills flow and singular Yang-Mills connections, Math. Ann., to appear.

[15] Huisken, G. Asymptotic behavior for singularities of the mean curvature flow, J. Differential Geom. 31, 285-299, (1990).

[16] Ilmanen, T. Elliptic regularization and partial regularity for motion by mean curvature, Mem. Amer. Math. Soc. \#520, (1994).

[17] Ilmanen, T. Singularities of mean curvature flow of surfaces, preprint, http://www. math.ethz.ch/ ilmanen/papers/pub.htm, (1995).

[18] Ilmanen, T. Lectures on mean curvature flow and related equations, Lecture Notes, ICTP, Trieste, http:// www. math. ethz.ch/ ilmanen/papers/pub.html, (1995).

[19] Ilmanen, T. Notes on mean curvature flow, in preparation, (2004).

[20] Li, P. and Yau, S.-T. On the parabolic kernel of the Schrödinger operator, Acta Math. 156, 153-201, (1986).

[21] Nash, J. Continuity of solutions of parabolic and elliptic equations, Amer. J. Math. 80, 935-954, (1958).

[22] Ni, L. The entropy formula for linear heat equation, J. Geom. Anal. 14(1), 87-100, (2004).

[23] Ni, L. Addenda to The entropy formula for linear heat equation, J. Geom. Anal. 14(2), 369-374, (2004).

[24] Ni, L. A new matrix Li-Yau-Hamilton inequality for Kahler-Ricci flow, submitted.

[25] Perelman, G. The entropy formula for the Ricci flow and its geometric applications, math.DG/ 0211159, November (2002).

[26] Perelman, G. Ricci flow with surgery on three-manifolds, math.DG/0303109, March (2003)

[27] Sesum, N. Limiting behavior of the Ricci flow, preprint, (2004).

[28] Uhlenbeck, K. Removable singularities in Yang-Mills fields, Comm. Math. Phys. 83, 11-29, (1982).

[29] Weinkove, B. A complex Frobenuis theorem, multiplier ideal sheaves and Hermitian-Einstein metrics on stable bundles, preprint, (2003).

[30] White, B. personal communication, (2000).

Received December 6, 2004

Department of Mathematics, University of Wisconsin, Madison WI 53706

Departement Mathematik, ETH Zentrum, 8092 Zürich, Switzerland

Department of Mathematics, University of California, San Diego, La Jolla CA 92093

e-mail: lni@math.ucsd.edu

Communicated by Peter Li 